\title{
Attenuated Live Virus Vaccine
}

National Cancer Institute

\section{Source}

National Cancer Institute. Attenuated Live Virus Vaccine. NCI Thesaurus. Code C1920.

Live vaccines prepared from microorganisms which have undergone physical adaptation (e.g., by radiation or temperature conditioning) or serial passage in laboratory animal hosts or infected tissue/cell cultures, in order to produce avirulent mutant strains capable of inducing protective immunity. Live-attenuated vaccines are used when a CD8+ T Cell (cellular cytotoxicity) response is desired. 